\title{
Effectiveness and Safety of Rivaroxaban in Patients With Cancer-Associated Venous Thrombosis
}

\author{
Christine G. Kohn, PharmDa; Gary H. Lyman, MDº Jan Beyer-Westendorf, MD, ; \\ Alex C. Spyropoulos, MD; ; Thomas J. Bunz, PharmD, PhD; William L. Baker, PharmD ${ }^{\text {a,s; }}$ \\ Daniel Eriksson, $\mathrm{MSc}^{\mathrm{h}}$; Anna-Katharina Meinecke, $\mathrm{PhD}^{\mathrm{h}}$; and Craig I. Coleman, PharmD ${ }^{\mathrm{a}, \mathrm{g}}$
}

\begin{abstract}
Background: Although not designated as guideline-recommended first-line anticoagulation therapy, patients are receiving rivaroxaban for the treatment and secondary prevention of cancer-associated venous thrombosis (CAT). We sought to estimate the cumulative incidence of recurrent venous thromboembolism (VTE), major bleeding, and mortality/hospice care in patients with CAT treated with outpatient rivaroxaban in routine practice. Methods: Using US MarketScan claims data from January 2012 through June 2015, we identified adults with active cancer (using SEER program coding) who had $\geq 1$ primary hospitalization or emergency department discharge diagnosis code for VTE (index event) and received rivaroxaban as their first outpatient anticoagulant within 30 days of the index VTE. Patients were required to have $\geq 180$ days of continuous medical/prescription benefits prior to the index VTE. Patients with a previous claim for VTE, atrial fibrillation, or valvular disease or receiving anticoagulation during the baseline period were excluded. We estimated the cumulative incidence with 95\% Cls of recurrent VTE, major bleeding, and mortality or need for hospice care at 180 days, assuming competing risks. Results: A total of 949 patients with active cancer were initiated on rivaroxaban following their index VTE. Time from active cancer diagnosis to index CAT was $\leq 90$ days for $27 \%$ of patients, 91 to 180 days for $19 \%$, and $>180$ days for $54 \%$. The mean [SD] age of patients was 62.5 [12.8] years, $43.6 \%$ had pulmonary embolism, and metastatic disease was present in $42.6 \%$. During follow-up, there were 37 cases of recurrent VTE, 22 cases of major bleeding (17 gastrointestinal, 3 intracranial, 1 genitourinary, and 1 other bleed), and 105 deaths/hospice claims. The cumulative incidence estimate was $4.0 \%(95 \% \mathrm{Cl}, 2.8 \%-5.4 \%)$ for recurrent $\mathrm{VTE}, 2.7 \%(95 \% \mathrm{Cl}, 1.7 \%-4.0 \%)$ for major bleeding, and $11.3 \%(95 \% \mathrm{Cl}$, $9.2 \%-13.6 \%)$ for mortality/hospice care. Conclusions: Event rates observed in this rivaroxaban-treated cohort were overall consistent with previous studies of patients with rivaroxaban- and warfarin-managed CAT.
\end{abstract}

J Natl Compr Canc Netw 2018;16(5):491-497 doi: 10.6004/jncen.2018.7008

Patients with active cancer have up to a 7 -fold increased risk of venous thromboembolism (VTE) compared with age-matched controls, corresponding to an

\footnotetext{
aHartford Hospital Evidence Based Practice Center, Hartford, Connecticut; ${ }^{\mathrm{b}}$ Fred Hutchinson Cancer Research Center, Seattle, Washington; 'Thrombosis Research Unit, Department of Medicine I, Division Hematology, University Hospital "Carl Gustav Carus" Dresden, Dresden, Germany; 'Kings Thrombosis Service, Department of Hematology, Kings College London, London, United Kingdom; 'Department of Medicine, Anticoagulation and Clinical Thrombosis Services, Northwell Health System, Hofstra Northwell School of Medicine, Manhasset, New York; ${ }^{\dagger}$ New England Health Analytics, LLC, Granby, Connecticut; 9University of Connecticut School of Pharmacy, Storrs, Connecticut; and 'Bayer AG, Berlin, Germany.
}

Submitted September 21, 2017; accepted for publication January 24, 2018.

Dr. Beyer-Westendorf has disclosed that he has received grant/research support from Bayer, Boehringer Ingelheim, Daiichi Sankyo, Pfizer, and Portola, and has served as a consultant to and has received honoraria from Bayer, Boehringer Ingelheim, Daiichi Sankyo, Pfizer, Portola, CSL Behring, and Medscape. Dr. Spyropoulos has disclosed that he has received grant/ research support from Janssen; has served as a consultant for Boehringer Ingelheim, Janssen, Bayer Healthcare, and Portola; and has served on annual incidence of approximately 1 thrombotic event per 200 patients with active cancer. ${ }^{1-3}$ Although anticoagulation is the cornerstone of management of cancer-

advisory committees for Boehringer Ingelheim and Janssen. Mr. Eriksson and Dr. Meinecke have disclosed that they are employees of Bayer AG. Dr. Coleman has disclosed that he received grant/research support from Bayer AG and Janssen Scientific Affairs, LLC, and that he has served as a consultant for Janssen Scientific Affairs and Bayer AG. The remaining authors have disclosed that they have no financial interests, arrangements, affiliations, or commercial interests with the manufacturers of any products discussed in this article or their competitors.

This study was supported by Bayer AG, Berlin, Germany. The sponsor had no role in study design; the collection, analysis, and interpretation of data; the writing of the report; or the decision to submit the article for publication.

Author contributions: Study concept and design: All authors. Data acquisition: All authors. Data analysis and interpretation: All authors. Manuscript preparation: All authors. Critical revision: All authors.

Correspondence: Craig I. Coleman, PharmD, University of Connecticut School of Pharmacy, 69 North Eagleville Road, Unit 3092, Storrs, CT 06269. Email: craig.coleman@hhchealth.org 
associated venous thrombosis (CAT), its use is complicated by patients' higher risk of both recurrent VTE and bleeding complications. ${ }^{3,4}$

Randomized controlled trials (RCTs) have demonstrated superior efficacy of continued low-molecular-weight heparins (LMWHs) over parenteral bridging to vitamin $\mathrm{K}$ antagonist (VKA) therapy for treatment and secondary prevention of CAT..$^{5-7}$ Consequently, current CAT treatment guidelines recommend LMWHs as first-line therapy, with oral anticoagulants reserved for patients unable or willing to use long-term parenteral agents. ${ }^{7-11}$ Despite CAT guideline recommendations, nearly one-fifth of patients receive rivaroxaban for acute treatment and secondary prevention of CAT in routine clinical practice. ${ }^{12}$

This claims database analysis sought to estimate the cumulative incidence of recurrent VTE, major bleeding, and mortality/hospice care in patients with CAT treated with rivaroxaban.

\section{Methods}

This article was written in compliance with the RECORD (REporting of studies Conducted using Observational Routinely-collected health Data) statement. ${ }^{13}$ We performed a retrospective, single-arm, administrative claims data-based analysis of patients with CAT treated with rivaroxaban as outpatients. Health insurance claims starting November 2012 (when rivaroxaban was approved for VTE treatment in the United States) through September 2015 (most recent data available at time of analysis) captured in the US Truven Health Analytics MarketScan Databases were used to perform this analysis. MarketScan combines 2 separate databases - a commercial and a Medicare supplemental database-to cover all age groups, and as of March 2017 contained claims from 260 contributing employers, 40 contributing health plans, and government and public organizations representing nearly 240 million covered American lives. ${ }^{14}$ MarketScan captures health plan enrollment records, demographics, ICD-9-CM diagnosis codes, procedure codes, admission and discharge dates, inpatient mortality data, outpatient medical services data, and prescription dispensing records. All data included in the MarketScan databases are deidentified and in compliance with the Health Insurance Portability and Accountability Act of 1996 to pre- serve participant anonymity and confidentiality. For this reason, the study was deemed exempt from Institutional Review Board oversight.

To be included in the current analysis, patients aged $\geq 18$ years had to have active cancer; $\geq 1$ primary discharge diagnosis code for deep vein thrombosis (DVT; ICD-9-CM codes 451.1, 451.2, 453.40, $453.41,453.42,453.8$, or 453.9 ) or pulmonary embolism (ICD-9-CM codes 415.1x); a positive predictive value for identifying any VTE of 95\% (95\% CI, 9397) ${ }^{15}$ linked to a hospital or emergency department stay; and be newly initiated on rivaroxaban within 30 days of hospitalization or emergency department visit for the index VTE event. Patients were required to be continuously enrolled in their health plan, with medical and pharmacy benefits for at least 180 days prior to the index event date (which serves as the study's baseline period). Patients with a VTE, atrial fibrillation, or valvular heart disease claim or a prescription claim for any anticoagulant during this baseline period were excluded. Patients with active cancer were identified using the NCI/Cancer Research Network algorithm, which uses ICD-9-CM diagnosis, Current Procedural Technology codes, Diagnosis-Related Group classification system, Healthcare Common Procedure Coding System codes, and National Drug Codes. ${ }^{16}$

Primary end points for this study were recurrent VTE (identified by the presence of an ICD-9-CM code, as detailed in the previous paragraph, in the primary discharge code associated with a subsequent hospital admission), major bleeding (defined as bleeding-related hospitalizations per the Cunningham algorithm), ${ }^{17}$ and all-cause mortality or a claim for hospice care in the 180 days following the index VTE event. Although in-hospital mortality data are reported in the MarketScan databases, out-of-hospital mortality data are not. For this study, we combined in-hospital death and need for hospice care without subsequent healthcare claims into a single end point. A similar approach was previously shown in claims data to have a positive predictive value of approximately $93 \%{ }^{18}$ for mortality, and is further supported by Mantha et al, ${ }^{19}$ who found that more than three-quarters of deaths in patients with CAT occurred during hospice care.

Patient baseline characteristics were determined based on claims during the 180 days preceding the index VTE event and were presented using descrip- 
Rivaroxaban for Cancer-Associated Venous Thrombosis

tive statistics. The presence of comorbidities and risk factors was determined using validated coding indices. ${ }^{16,20,21}$ Categorical variables were summarized as proportions. Continuous variables were summarized as mean [SD] or medians and interquartile ranges (IQRs). Cumulative incidence estimates for recurrent VTE, major bleeding, and mortality/hospice care were calculated, along with 95\% CIs, assuming competing risks (for recurrent VTE and major bleeding), using the method described by Fine and Gray, ${ }^{22}$ and graphically depicted as time-to-event curves. An intent-to-treat approach was used and patients were followed until a primary end point, loss to follow-up, or end-of-study period defined as 180 days post-index VTE to match ongoing randomized trials of rivaroxaban in $\mathrm{CAT}^{10}{ }^{10}$ Cumulative incidence estimates per cancer types constituting at least $10 \%$ of the study population were also calculated. Database management and statistical analysis was performed using SAS 9.4 (SAS Institute Inc.) and R version 3.1.2 (The R Foundation for Statistical Computing) with the accompanying cmprsk package. ${ }^{23}$

\section{Results}

A total of 949 patients with active cancer and a primary diagnosis of VTE were initiated on outpatient anticoagulation therapy with rivaroxaban within 30 days of the index event and included in this analysis. Baseline demographics, comorbidities, and medication use are reported in Tables 1-3. The median time from index VTE to first outpatient rivaroxaban fill was 2 days (IQR, 1, 5), and median time between first and last rivaroxaban prescription was 114 days (IQR, 47, 180). The mean age of identified patients was 62.5 [12.8] years, $45.6 \%$ were men, and pulmonary embolism $( \pm$ DVT) was present in $43.6 \%$. Time from the diagnosis of active cancer to index CAT was $\leq 90$ days in $27 \%$ of patients, 91 to 180 days for $19 \%$, and $\geq 181$ days for $54 \%$. Cancer sites (not limited to one per patient) for this rivaroxaban-treated cohort were as follows: $25.5 \%$ breast, $21.0 \%$ genitourinary (including prostate), $17.3 \%$ gastrointestinal, $13.2 \%$ hematologic, $11.5 \%$ lung, $7.4 \%$ gynecologic, $5.8 \%$ pancreas, and $39.5 \%$ other. Metastatic disease was present in $42.6 \%$ of patients.

During the 6-month follow-up period, there were 37 cases of recurrent VTE, 22 cases of major bleeding (17 gastrointestinal, 3 intracranial, 1 geni-

\begin{tabular}{|lc|}
\hline Table 1. Baseline Demographics & \\
\hline Variable & $\begin{array}{c}\text { Total Cohort, } \mathbf{n}(\%) \\
\mathrm{N}=949\end{array}$ \\
\hline \begin{tabular}{l} 
Mean age [SD], y \\
\hline$>65 \mathrm{y}$
\end{tabular} & $62.5(12.8)$ \\
\hline Male sex & $361(38.0)$ \\
\hline \begin{tabular}{l} 
Year of inclusion \\
\hline 2012
\end{tabular} & $433(45.6)$ \\
\hline $\begin{array}{l}\text { 2013 } \\
\text { 2014 }\end{array}$ & $14(1.5)$ \\
\hline $\begin{array}{l}\text { 2015 } \\
\text { Insurance plan type }\end{array}$ & $294(31.0)$ \\
\hline $\begin{array}{l}\text { HMO } \\
\text { POS }\end{array}$ & $482(50.8)$ \\
\hline \begin{tabular}{l} 
PPO \\
\hline Other
\end{tabular} & $159(16.8)$ \\
\hline $\begin{array}{l}\text { VTE type } \\
\text { DVT only }\end{array}$ & $50(9.3)$ \\
\hline $\begin{array}{l}\text { PE ( } \pm \text { DVT) } \\
\text { Time from index event to first } \\
\text { anticoagulant claim, d } \\
\text { (median, IQR) }\end{array}$ & $528(55.6)$ \\
\hline $\begin{array}{l}\text { Time between first and last } \\
\text { rivaroxaban prescription, d } \\
\text { (median, IQR) }\end{array}$ & $280(29.5)$ \\
\hline $\begin{array}{l}\text { Mean [SD] number of } \\
\text { hospitalizations during previous } \\
180 \text { days }\end{array}$ & $114(47,180)$ \\
\hline
\end{tabular}

Abbreviations: DVT, deep vein thrombosis; $\mathrm{HMO}$, health maintenance organization; IQR, interquartile range; PE, pulmonary embolism; POS, point of service; PPO, preferred provider organization; VTE, venous thromboembolism.

tourinary, and 1 other bleed), and 105 deaths or hospice claims ( 0.42 of which occurred during a hospital stay). The 180-day cumulative incidence estimate was $4.0 \%$ (95\% CI, 2.8\%-5.4\%) for recurrent VTE, $2.7 \%$ (95\% CI, 1.7\%-4.0\%) for major bleeding, and $11.3 \%$ (95\% CI, 9.2\%-13.6\%) for mortality/hospice care (Table 4). The cumulative incidence time-toevent curves assuming competing risk for each end point are depicted in Figure 1. Cumulative incidence estimates for recurrent VTE, major bleeding, and mortality/hospice care for each cancer type present in $\geq 10 \%$ of our study population are reported in Table 4.

\section{Discussion}

Current recommended first-line treatment for the management of CAT is a LMWH.9,11,24,25 This rec- 
Kohn et al

\begin{tabular}{|c|c|}
\hline Variable & $\begin{array}{l}\text { Total Cohort, } \mathrm{n}(\%) \\
\mathrm{N}=949\end{array}$ \\
\hline $\begin{array}{l}\text { Mean [SD] number of Elixhauser } \\
\text { comorbidities }\end{array}$ & $14.29(9.95)$ \\
\hline 1 & $73(7.7)$ \\
\hline 2 & $123(13.0)$ \\
\hline 3 & $121(12.8)$ \\
\hline$\geq 4$ & $632(66.6)$ \\
\hline \multicolumn{2}{|l|}{ Cancer type } \\
\hline Breast & $242(25.5)$ \\
\hline Genitourinary (including prostate) & $199(21.0)$ \\
\hline Gastrointestinal & $164(17.3)$ \\
\hline Colorectal & $125(13.2)$ \\
\hline Stomach/Esophagus & $39(4.1)$ \\
\hline Hematologic & $125(13.2)$ \\
\hline Lung & $109(11.5)$ \\
\hline Gynecologic & $70(7.4)$ \\
\hline Pancreas & $55(5.8)$ \\
\hline Other & $375(39.5)$ \\
\hline Metastatic disease & $404(42.6)$ \\
\hline Inferior vena cava filter & $19(2.0)$ \\
\hline Central venous catheter & $47(5.0)$ \\
\hline \multicolumn{2}{|l|}{ Chronic kidney disease } \\
\hline Stage $1-2$ & $28(3.0)$ \\
\hline Stage 3 & $56(5.9)$ \\
\hline Stage 4-5 & $18(1.9)$ \\
\hline Unstaged & $49(5.2)$ \\
\hline Renal dialysis & $2(0.2)$ \\
\hline Coagulopathy & $93(9.8)$ \\
\hline Obesity & $182(19.2)$ \\
\hline Weight loss & $106(11.2)$ \\
\hline Paralysis & $27(2.8)$ \\
\hline Tobacco use & $186(19.6)$ \\
\hline Varicose veins & $17(1.8)$ \\
\hline Hip or pelvis fracture & $29(3.1)$ \\
\hline Chronic lung disease & $245(25.8)$ \\
\hline Pneumonia & $151(15.9)$ \\
\hline Hypertension & $591(62.3)$ \\
\hline Diabetes mellitus & $243(25.6)$ \\
\hline Hyperlipidemia & $377(39.7)$ \\
\hline $\begin{array}{l}\text { Rheumatoid arthritis/collagen vascular } \\
\text { disease }\end{array}$ & $45(4.7)$ \\
\hline Heart failure & $93(9.8)$ \\
\hline Depression & $122(12.9)$ \\
\hline Psychosis & $47(5.0)$ \\
\hline Liver dysfunction & $42(4.4)$ \\
\hline Hypothyroidism & $158(16.6)$ \\
\hline Anemia & $282(29.7)$ \\
\hline Alcohol abuse & $24(2.5)$ \\
\hline Drug abuse & $10(1.1)$ \\
\hline Ischemic stroke & $40(4.2)$ \\
\hline Transient ischemic attack & $16(1.7)$ \\
\hline Acute coronary syndrome & $34(3.6)$ \\
\hline Inflammatory bowel disease & $13(1.4)$ \\
\hline
\end{tabular}

\begin{tabular}{|lc|}
\hline Table 3. Baseline Medication Use & $\begin{array}{c}\text { Total Cohort, } \mathbf{n}(\%) \\
\mathbf{N}=949\end{array}$ \\
\hline Estrogens & $31(3.3)$ \\
\hline Erythropoietin-stimulating agents & $11(1.2)$ \\
\hline Antiplatelets or NSAIDs & $235(24.8)$ \\
\hline ACE inhibitors or ARBs & $338(35.6)$ \\
\hline$\beta$-blockers & $246(25.9)$ \\
\hline Calcium channel blockers & $171(18.0)$ \\
\hline Diuretics & $223(23.5)$ \\
\hline Digoxin & $11(1.2)$ \\
\hline Statin & $288(30.3)$ \\
\hline Insulin & $51(5.4)$ \\
\hline Noninsulin diabetes drugs & $147(15.5)$ \\
\hline Antidepressants & $265(27.9)$ \\
\hline Antiulcer drugs & $310(32.7)$ \\
\hline
\end{tabular}

Abbreviations: ACE, angiotensin-converting enzyme; ARBs,

angiotensin receptor blockers; NSAIDs, nonsteroidal anti-inflammatory drugs.

ommendation is based on RCT data showing a lower risk of recurrent VTE and similar bleeding risk with LMWH compared with VKA therapy. ${ }^{26}$ Guidelines from ASCO, British Committee for Standards in Haematology (BCSH), American College of Chest Physicians (ACCP), and the International Initiative on Thrombosis and Cancer (ITAC-CME) suggest that oral anticoagulants can be considered an option for long-term CAT treatment based on patient preference or if LMWHs are contraindicated or unavailable. ${ }^{9,11,24,25}$ However, there is no consensus on the role of non-VKA oral anticoagulants, such as rivaroxaban, for the management of CAT. ASCO guidelines ${ }^{9}$ recommend against their use, citing insufficient evidence, whereas ITAC-CME guidance says they can be considered in patients not receiving systemic anticancer therapy when other alternatives are not preferred or available. ${ }^{25}$ The 2016 ACCP guidelines do not provide a preference between VKA and non-VKA oral anticoagulants in patients not receiving a LMWH. ${ }^{11}$ Regardless of these recommendations, rivaroxaban was used in 19\% of patients in 2015 according to a retrospective evaluation of US prescription patterns for CAT. ${ }^{12}$ Thus, there remains a great need for additional evidence supporting its use in this population.

Our retrospective administrative claims database study reports on outcomes for $>900$ patients with active cancer experiencing a VTE and treated 
Rivaroxaban for Cancer-Associated Venous Thrombosis

Table 4. Six-Month Cumulative Incidence Estimates

\begin{tabular}{|lccc|} 
Cancer Type & $\begin{array}{c}\text { Recurrent VTE, \% } \\
(95 \% \text { Cl) }\end{array}$ & $\begin{array}{c}\text { Major Bleeding, \% } \\
(95 \% \text { Cl) }\end{array}$ & $\begin{array}{c}\text { Mortality/Hospice, \% } \\
(95 \% \text { Cl) }\end{array}$ \\
\hline Full cohort & $4.0(2.8-5.4)$ & $2.7(1.7-4.0)$ & $11.3(9.2-13.6)$ \\
\hline Breast & $5.0(2.9-8.5)$ & $2.1(0.9-4.7)$ & $10.3(7.1-14.8)$ \\
\hline Genitourinary (including prostate) & $2.5(1.1-5.8)$ & $1.0(0.3-3.6)$ & $8.0(5.0-12.7)$ \\
\hline Gastrointestinal & $3.7(1.7-7.8)$ & $6.1(3.4-10.9)$ & $13.4(9.0-19.5)$ \\
\hline Hematologic & $1.6(0.4-5.7)$ & $0.8(0.1-4.4)$ & $4.8(2.2-10.1)$ \\
\hline Lung & $6.4(3.2-12.7)$ & $3.7(1.4-9.1)$ & $24.8(17.6-33.6)$ \\
\hline
\end{tabular}

Abbreviation: VTE, venous thromboembolism.

${ }^{a}$ Reported cancer types present in at least $10 \%$ of the study cohort.

as outpatients with rivaroxaban, making it the largest assessment of rivaroxaban for CAT published to date and allowing for analysis by active cancer subtypes. During the 6-month intent-to-treat follow-up period, we observed overall incidences of recurrent VTE and major bleeding consistent with those reported in prior prospective observational studies of rivaroxaban in CAT. ${ }^{19,27}$ Mantha et al ${ }^{19}$ conducted a single-arm prospective cohort study of 200 patients with CAT, showing a 6-month cumulative incidence of recurrent VTE and major bleeding with rivaroxaban of $4.4 \%$ (95\% CI, 1.4\%-7.4\%) and $2.2 \%(95 \%$ CI, 0\%-4.2\%), respectively. These clinical events were identified through a search of patient clinical notes. In a subset of 146 patients with cancer identified from the multicenter, prospective, noninter-

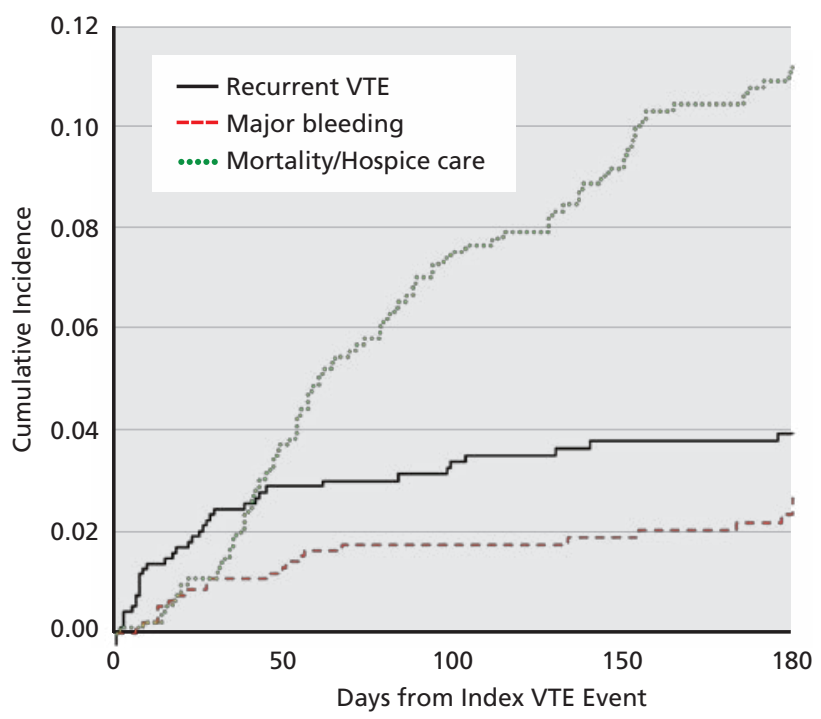

Figure 1. Cumulative incidence time-to-event curves for recurrent venous thromboembolism, major bleeding, and mortality/hospice care. For this analysis, the time to death/hospice care was assigned as the date of in-hospital death or last date of service associated with the hospice claim. ventional XALIA study, VTE recurrence and major bleeding rates of $3.4 \%(95 \% \mathrm{CI}, 1.1 \%-7.8 \%)$ and $1.4 \%$ (95\% CI, 0\%-4.9\%), respectively, were reported. ${ }^{26}$ Unlike the study by Mantha and colleagues, end points in XALIA were adjudicated by a masked central committee.

Our results are also in line with rivaroxaban data from RCTs. ${ }^{28}$ In 354 patients diagnosed with cancer at baseline or during anticoagulation treatment and randomized to receive rivaroxaban $(15 \mathrm{mg}$ twice daily for 21 days followed by $20 \mathrm{mg}$ daily) in the pooled EINSTEIN trial population, the incidence of recurrent VTE was $5 \%$ and the incidence of major bleeding was $2 \%$ over a mean follow-up of 6 months. ${ }^{28}$ Of note, the patients with CAT who received rivaroxaban did not demonstrate a higher rate of recurrent VTE (hazard ratio [HR], 0.67; 95\% CI, 0.35-1.30) and exhibited less major bleeding (HR, 0.42; 95\% CI, 0.18-0.99) compared with those treated with enoxaparin and bridged to VKA.

Certain types of cancers are known to be associated with higher incidence of VTE recurrence, including lung and gastrointestinal malignancies ${ }^{29,30}$; conversely, breast cancer and hematologic malignancies are associated with lower recurrent VTE risks. Therefore, it is important to consider the distribution of cancer types when evaluating VTE event rates in CAT studies. The prevalence of lung and gastrointestinal cancer in the current study was $11.5 \%$ and $17.3 \%$, respectively, compared with $11.5 \%$ and $12 \%$ in the study by Mantha et al, ${ }^{19} 3.4 \%$ and $13.7 \%$ in XALIA, ${ }^{27}$ and $7.4 \%$ and $14.3 \%$ in the rivaroxaban arm of EINSTEIN. ${ }^{28}$ The relative similarities in overall prevalence of these higher-risk cancer types support the comparable rates of rivaroxaban-associated recurrent VTE observed. 
Incidence of the pooled mortality/hospice end point with rivaroxaban in our study was $11.3 \%$. This varies somewhat from the approximate 6-month mortality rates reported in rivaroxaban-treated patients in the study by Mantha et $\mathrm{al}^{19}(17.6 \%)$, the subgroup of patients with cancer in the XALIA study $(4.8 \%), 27$ and the subgroup of patients with active cancer in EINSTEIN ${ }^{28}$ (17\%). The comparatively large variation in mortality in these different CAT trials may be partly explained by differences in study methodology, but is most likely driven by differences in study population. Approximately $90 \%$ of deaths seen in CAT studies are due to cancer progression rather than complications of recurrent VTE. ${ }^{3}$ Furthermore, data suggest that rivaroxaban is more likely to be used in less-ill patients with CAT. Of the 557 patients in XALIA who had active cancer at baseline, $26.2 \%$ were prescribed rivaroxaban, with the rest receiving standard therapy. The incidence of mortality in these patients (not controlled for differences in patient characteristics) was $4.8 \%$ in the rivaroxaban group and $24.7 \%$ in the LMWH group. ${ }^{27}$

There are some limitations related to the retrospective claims database nature of our analysis that deserve mention. Misclassification (measurement error) and selection bias (selection of patients to receive a treatment in a nonrandomized fashion) may affect the internal and external validity of our study. ${ }^{31}$ In addition, information on cancer staging and patient functional status are either unavailable or not well-defined in claims data, which prevented us from describing this CAT population in greater detail. For these reasons, and because of the relatively small sample of patients receiving rivaroxaban in our data set, we opted not to perform a comparative analyses of rivaroxaban versus LMWH or warfarin. Our study relied on claims contained within the Truven MarketScan databases, ${ }^{14}$ which include data on commercially insured people (with employer-sponsored health plans) or those with Medicare supplemental plans. Therefore, our study may not be generalizable to the US population as a whole. Moreover, the MarketScan databases do not report on out-ofhospital death. The need for us to use hospice care as a surrogate for out-of-hospital death ${ }^{18}$ along with the fact that we followed patients beginning with their first outpatient prescription for rivaroxaban (and not evaluating outcomes during the index VTE encounter) may have resulted in an underestimation of mortality risk in our study.

\section{Conclusions}

The cumulative recurrent VTE and major bleeding event rates of $4.0 \%$ and $2.7 \%$, respectively, observed in this rivaroxaban-treated cohort were relatively consistent with those observed in previous studies of rivaroxaban. Our cumulative mortality/hospice rate of approximately $11 \%$ was lower than mortality rates observed in some previous CAT studies, perhaps as an artifact of our study population or methodology, and therefore should be interpreted with caution. Once completed, the randomized multicenter SELECT-D trial (ISRCTN86712308) will provide additional data regarding the use of rivaroxaban in patients with CAT, including data on its comparative efficacy and safety compared with the LMWH dalteparin. ${ }^{10}$

\section{References}

1. Heit JA, Silverstein MD, Mohr DN, et al. Risk factors for deep vein thrombosis and pulmonary embolism. Arch Intern Med 2000;160:809_ 815 .

2. Bloom JW, Doggen CJM, Osanto S, et al. Malignancies, prothrombotic mutations and the risk of venous thrombosis. JAMA 2005;293:715-722.

3. Lee AY, Levine MN, Baker RI, et al; for the Randomized Comparison of Low-Molecular-Weight Heparin Versus Oral Anticoagulant Therapy for the Prevention of Recurrent Venous Thromboembolism in Patients With Cancer Investigators. Low-molecular-weight heparin versus a coumarin for the prevention of recurrent venous thromboembolism in patients with cancer. N Engl J Med 2003;349:146-153.

4. Prandoni $\mathrm{P}$, Lensing AW, Piccioli A, et al. Recurrent venous thromboembolism and bleeding complications during anticoagulant treatment in patients with cancer and venous thromboembolism. Blood 2002;100:3484-3488.

5. Lee AY, Levine MN. Venous thromboembolism and cancer: risks and outcomes. Circulation 2003;107(Suppl 1):I17-21.

6. Hull RD, Pineo GF, Brant RF, et al; LITE Trial Investigators. Long term low-molecular-weight heparin versus usual care in proximal-vein thrombosis patients with cancer. Am J Med 2006;119:1062-1072.

7. Lee AY, Kamphuisen PW, Meyer G, et al. Tinzaparin vs warfarin for treatment of acute venous thromboembolism in patients with active cancer: a randomized clinical trial. JAMA 2015;314:677-686.

8. Lee AY, Peterson EA. Treatment of cancer-associated thrombosis. Blood 2013;122:2310-2317.

9. Lyman GH, Bohlke K, Khorana AA, et al; American Society of Clinical Oncology. Venous thromboembolism prophylaxis and treatment in patients with cancer: American Society of Clinical Oncology clinical practice guideline update 2014. J Clin Oncol 2015;33:654-656.

10. Bach M, Bauersachs R. Spotlight on advances in VTE management: CALLISTO and EINSTEIN CHOICE. Thromb Haemost 2016;116:S2432.

11. Kearon C, Akl EA, Ornelas J, et al. Antithrombotic therapy for VTE disease: chest guideline and expert panel report. Chest 2016;149:315-352. 
12. Khorana AA, Yannicelli D, McCrae KR, et al. Evaluation of US prescription patterns: are treatment guidelines for cancer-associated venous thromboembolism being followed? Thromb Res 2016;145:51-53.

13. Benchimol EI, Smeeth L, Guttmann A, et al. The Reporting of studies Conducted using Observational Routinely-collected health Data (RECORD) statement. PLoS Med 2015;12:e1001885.

14. Hansen L. The Truven Health MarketScan Databases for life sciences researchers. Available at: https://truvenhealth.com/Portals/0/Assets/2017MarketScan-Databases-Life-Sciences-Researchers-WP.pdf. Accessed October $8,2017$.

15. White RH, Garcia M, Sadeghi B, et al. Evaluation of the predictive value of ICD-9-CM coded administrative data for venous thromboembolism in the United States. Thromb Res 2010;126:61-67.

16. National Cancer Institute, Cancer Research Network (U24 CA171524). Cancer Therapy Look-up Tables. Available at: https://crn.cancer.gov/ resources/codes.html. Accessed September 5, 2017.

17. Cunningham $\mathrm{A}$, Stein $\mathrm{CM}$, Chung $\mathrm{CP}$, et al. An automated database case definition for serious bleeding related to oral anticoagulant use. Pharmacoepidemiol Drug Saf 2011;20:560-566.

18. Ooba N, Setoguchi S, Ando T, et al. Claims-based definition of death in Japanese claims database: validity and implications. PLoS One 2013;8:e66116.

19. Mantha S, Laube E, Miao Y, et al. Safe and effective use of rivaroxaban for treatment of cancer-associated venous thromboembolic disease: a prospective cohort study. J Thromb Thrombolysis 2017;43:166-171.

20. Moore $B J$, White $S$, Washington $R$, et al. Identifying increased risk of readmission and in-hospital mortality using hospital administrative data: the AHRQ Elixhauser Comorbidity Index. Med Care 2017;55:698-705.

21. Centers for Medicare \& Medicaid Services. Chronic Conditions Data Warehouse. Chronic Condition Categories. Available at: https://www. ccwdata.org/web/guest/condition-categories. Accessed November 8, 2017.

22. Fine JP, Gray RJ. A proportional hazards model for the subdistribution of a competing risk. J Am Stat Assoc 1999;94:496-509.
23. Package 'cmprsk': Subdistribution Analysis of Competing Risks. Available at: https://cran.r-project.org/web/packages/cmprsk/cmprsk.pdf. Accessed September 5, 2017.

24. Lyman GH, Khorana AA, Kuderer NM, et al. Venous thromboembolism prophylaxis and treatment in patients with cancer: American Society of Clinical Oncology clinical practice guideline update. J Clin Oncol 2013;31:2189-2204.

25. Farge $D$, Bounameauxz $H$, Brenner $B$, et al. International clinical practice guidelines including guidance for direct oral anticoagulants in the treatment and prophylaxis of venous thromboembolism in patients with cancer. Lancet Oncol 2016;17:e452-466.

26. Posch F, Konigsbrugge O, Zielinski C, et al. Treatment of venous thromboembolism in patients with cancer: a network meta-analysis comparing efficacy and safety of anticoagulants. Thromb Res 2015;136:582-589.

27. Ageno W, Mantovani LG, Haas S, et al. Subgroup analysis of patients with cancer in XALIA: a noninterventional study of rivaroxaban versus standard anticoagulation for VTE. TH Open 2017;1:e33-42.

28. Prins $M H$, Lensing AW, Brighton $T A$, et al. Oral rivaroxaban versus enoxaparin with vitamin $\mathrm{K}$ antagonist for the treatment of symptomatic venous thromboembolism in patients with cancer (EINSTEIN-DVT and EINSTEIN-PE): a pooled subgroup analysis of two randomized controlled trials. Lancet Haematol 2014;1:e37-46.

29. Louzada ML, Majeed H, Dao V, Wells PS. Risk of recurrent venous thromboembolism according to malignancy characteristics in patients with cancer-associated thrombosis: a systematic review of observational and interventional studies. Blood Coagul Fibrinolysis 2011;22:86-91.

30. Louzada ML, Carrier M, Lazo-Langner A, et al. Development of a clinical prediction rule for risk stratification of recurrent venous thromboembolism in patients with cancer-associated venous thromboembolism. Circulation 2012;126:448-454

31. Gandhi SK, Salmon W, Kong SZ, Zhao SZ. Administrative databases and outcomes assessment: an overview of issues and potential utility. J Manag Care Spec Pharm 1999;5:215-222.

\section{Register now at NCCN.org/academy}

NCCN Academy for Excellence \& Leadership in Oncology ${ }^{\mathrm{TM}}$ School of Pharmaceutical \& Biotech Business

\section{Tuesday, July 10, 2018 • 8:45 AM - 2:15 PM The Rittenhouse • Philadelphia, Pennsylvania \\ (Breakfast and registration begin at 8:00 AM)}

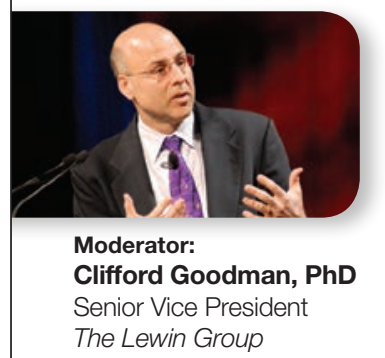

Module I: Who Exactly is the Payer? A View across the Oncology Ecosystem

Module II: Meet the NCCN Guidelines ${ }^{\circledR}$ Panel Experts

Module III: Incorporating the Patient Voice in Shared Decision Making

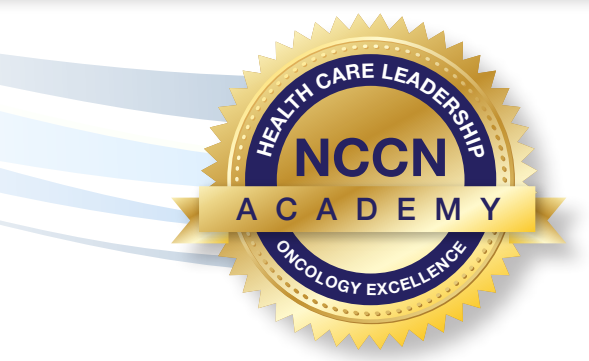

\section{Celebrating 10 years!}

By attending, professionals will be able to:

- Hear directly from NCCN Guidelines ${ }^{\circledR}$ Panel Members;

- Improve working knowledge of key business, policy, coverage, reimbursement, informational, and operational issues in oncology;

- Gather valuable insights into developing effective strategies for navigating the various constituencies in cancer care.

Visit NCCN.org/academy to enroll and to access the complete program curriculum. 